\title{
Laser Scanning Confocal Microscope-Based Fluorescence Recovery After Photobleaching (FRAP) Models for Pure Diffusion and Binding Diffusing Kinetics on Various Geometries in 1-3 Spatial Dimensions
}

\author{
M. Kang, ${ }^{*}$ E. DiBenedetto, ${ }^{* * *}$ and A. K. Kenworthy**** \\ * Department of Molecular Physiology and Biophysics, Vanderbilt University School of Medicine, \\ Nashville, TN 37232 \\ ** Department of Mathematics, Vanderbilt University, Nashville, TN 37240 \\ *** Department of Cell and Developmental Biology, Vanderbilt University School of Medicine, \\ Nashville, TN 37232
}

Diffusion and binding-diffusion are ubiquitous processes with importance across many scales of biology. Many cellular events depend strongly on the diffusivity and binding rates of proteins and other biomolecules, which are characterized by a diffusion coefficient (D) and binding rate constants $\left(\mathrm{k}_{\mathrm{on}}, \mathrm{k}_{\mathrm{off}}\right)$. Therefore, determining the kinetic rate constants such as diffusion coefficients or binding rate constants of proteins is important to understand cell function.

FRAP has been a useful technique for studying reaction-diffusion kinetics of biomolecules in cells since its development in the 1970s [1]. Classically, FRAP measurements were performed using a focused, static laser beam to bleach molecules. In recent years, commercial laser scanning confocal microscopes (LSCMs) have become widely utilized to study of intracellular protein dynamics $[2,3,4]$. As such, confocal FRAP can now be used to study binding-diffusion kinetics of proteins within their native environments, an important goal in light of the universality of reaction and diffusion processes in cells.

In spite of the popularity of LSCM based FRAP (confocal FRAP), most FRAP models available in the literature were developed for the static laser beam based conventional FRAP approaches, which are based on two critical assumptions: 1) the bleaching time scale is much faster than protein kinetic scales so that immediate photobleaching can be assumed; and 2) the bleach region of interest (ROI) is small enough so that the compartments from which recovery occurs (for example the plasma membrane or the cytoplasm) can be considered as infinite 2D and 3D spaces.

However, the $1^{\text {st }}$ assumption does not necessarily apply in confocal FRAP experiments, especially for soluble proteins, since in laser scanning confocal microscopes using a single laser for bleaching and imaging, the time required to scan the bleach region can allow for diffusion during the bleach [5]. Due to the long scanning time of confocal laser scanning microscopes, conventional FRAP analysis applied to confocal FRAP provides confounding kinetic parameter values [5]. To address this intrinsic property of confocal FRAP, we recently developed a formalism that can be used to correct for binding and/or diffusion during the photobleaching event and showed that this is a critical consideration in confocal FRAP analysis [4,5].

The $2^{\text {nd }}$ assumption is also not legitimate in many cases and geometric consideration is required depending on the locations in cells where FRAP experiments are performed. Although various versions of FRAP models have been developed in the literature, to our knowledge the consequences 
of these two assumptions for both pure diffusion and binding-diffusion kinetics have not been systematically evaluated. To this end, FRAP models for pure diffusion and binding diffusing kinetics (for either immobile or mobile bound species) were formulated for various geometries in 1-3 spatial dimensions with different boundary conditions, which are applicable to both conventional and confocal FRAP.

For a circular bleaching spot, the geometries considered in this study are 1) the infinite line (R1), 2) a finite interval $([-1,1]), 3)$ a circle $(\mathrm{S} 1), 4)$ the infinite plane $(\mathrm{R} 2), 5)$ a rectangle $([-1 \mathrm{x}, 1 \mathrm{x}] \times[-1 \mathrm{y}, 1 \mathrm{y}])$ 6) a disc (D2), 7) a sphere (S2), and 8) the infinite three dimensional space (R3) for reflective and restrictive boundary conditions. The models are summarized in Table 1.

TABLE 1. FRAP models derived in this study

\begin{tabular}{llccc}
\hline \multicolumn{1}{c}{ Domain } & Boundary Conditions & Free Diffusion & \multicolumn{2}{c}{ Binding Diffusion } \\
\cline { 4 - 5 } & & & $\mathrm{D}_{1}, \mathrm{D}_{2}>0$ & $\mathrm{D}_{1}>0, \mathrm{D}_{2}=0$ \\
\hline Infinite line & N/A & $\sqrt{ }$ & $\sqrt{ }$ & $\sqrt{ }$ \\
Finite interval & Neumann/Dirichlet & $\sqrt{ }$ & $\sqrt{ }$ & $\sqrt{ }$ \\
Circle & N/A & $\sqrt{ }$ & $\sqrt{ }$ & $\sqrt{ }$ \\
Infinite plane & N/A & $\sqrt{ }$ & $\sqrt{ }$ & $\sqrt{ }$ \\
Rectangle & Neumann/Dirichlet & $\sqrt{ }$ & $\sqrt{ }$ \\
Disc & Neumann/Dirichlet & $\sqrt{ }$ & $\sqrt{ }$ \\
Sphere & N/A & $\sqrt{ }$ & $\sqrt{ }$ \\
Infinite 3D space & N/A & $\sqrt{ }$ & & $\sqrt{ }$ \\
\hline
\end{tabular}

References

[1] D. Axelrod et al., Biophys J. 16 (1976) 1055.

[2] G.E. Carrero et al., Bull Math Biol. 66 (2004) 1515.

[3] B.L. Sprague ei al., Biophys J. 86 (2004) 3473.

[4] M. Kang et al., Biophys J. 99 (2010) 2737.

[5] M. Kang et al., Biophys J. 97 (2009) 1501.

[6] This research was supported by R01 GM073846 and NSF/DMS 0970008. 